\title{
Determination of Mental Stress Effects on the Cognitive Function in Medical Students of LUMHS at the time of Examination
}

\author{
Shafaq Ansari, Farheen Shaikh, Tazeen Shah
}

ABSTRACT

BACKGROUND: Cognition function refers to memory, the ability to learn new information, language, and reading conception. Mental stress may disrupt cognitive function. The medical students are exposed frequently to emotional disturbance and stress and these may adversely affect the cognition.

OBJECTIVE: To analyse the extent of impairment of cognitive function due to mental stress during examination of medical students by implicates the application of Stroop test.

STUDY DESIGN: Cross sectional comparative study

SETTING: The study was conducted at the Department of Physiology LUMHS Jamshoro over 6 months duration from August 2015 to February 2016.

METHODOLOGY: The volunteer information brochures / leaflets were posted on the main notice board of the LUMHS campus. Total 150 participants $(n=100$ having their examination within a week, $n=50$ not preparing for examination) were selected by simple random sampling technique. All four test of Stroop test were performed (Power lab T-125 AD-instrument Australia) and time taken by participants were noted and compared between both groups.

RESULTS: Breathing rate, heart rate, systolic and diastolic blood pressure (BP) showed statistically significant differences $(p<0.05)$ between two groups. The differences in the results of all four test for Stroop effect; Monochrome reading, color naming, reading color words and color naming were highly significant between those having their examination ahead (group A) within one week and those having no examination ahead (group B). Congruent Stroop test - exercise 1 and 2 showed significant differences for time domain per single word in group A and B respectively. Incongruent Stroop test showed time per single word of 2.3000 vs. 1.7500 seconds in exercise 3 (t-value 23.13, $p$-value 0.0001 ). Time taken per single word was noted as 2.1000 vs. 1.6100 seconds in exercise 3 (t-value 16.56, p-value 0.0001).

CONCLUSION: As the results showed increased reaction time and interferences for Stroop effect among medical students under examination stress, it may be concluded that the mental stress adversely affects the cognitive functions.

KEY WORDS: Stroop test, Mental stress, Cognitive functions, Medical student.

This article may be cited as: Ansari S, Shaikh F, Shah T. Determination of Mental Stress Effects on the Cognitive Function in Medical Students of LUMHS at the time of Examination. J Liaquat Uni Med Health Sci. 2018;17(01):23-8. doi: 10.22442/jlumhs.181710544

\section{INTRODUCTION}

Cognition is the set of all mental abilities and processes related to knowledge and it is also known that there is high frequency of emotional disturbance and stress in medical students as compared to that in the general population ${ }^{1}$. It is usually observed that medical students undergo remarkable stress during various stages of their course ${ }^{2}$. High level of stress may have a negative impact on cognitive function and learning potential of the students ${ }^{3}$. Medical students deal with various stressors that are peculiar to a medical school, e.g. academic overwork, financial worries, lack of free time, pressures to meet dead-lines, work place relationships and career choices ${ }^{4}$. Previous studies have suggested that stress -related factors may be interlinked, forming a complex psychological structure that may exert effects on academic performance of medical students ${ }^{5}$. The individual respond to stress in many ways that can affect the person as well as the environment. Evidence suggests that female medical students may suffer from low self-confidence and increased anxiety especially the time when facing examiner during examination ${ }^{6}$. The examinations for a medical student are supposed to be as high stressor, much more in females ${ }^{7}$. In many medical universities, academic environment itself is stressful that encourages competition rather than collaboration between learners ${ }^{8}$.

The American Institute of Stress defines mental stress as "physical, mental, or emotional strain or tension as well as a condition or feeling experienced when a 
person perceives that demands exceed the personal and social resources the individual is able to mobilize"9.

Individual response to stress manifests itself positively or negatively i.e., positive stress gives stimulus that helps to drive an individual to meet goals. It also helps in adaptation and reacting positively to the environment and environmental change. The negative stress may produce many harmful effects that if un-controlled may lead to serious illnesses such as, myocardial ischemia, arrhythmias and left ventricular dysfunction. This takes place due to stress induced activation of Sympathetic Nervous System (SNS) resulting in increased heart rate $(\mathrm{HR})$ and blood pressure (BP), creating extra myocardial oxygen consumption and demand. ${ }^{10}$ This can also lead to a decreased ability to concentrate, a loss of attention and focus, and increased anxiety and tension ${ }^{11}$.

Cognitive function refers to an individual's capability to process thoughts. Cognition mainly refers to memory, the ability to learn new information, language, and reading conception.

The stress affects cognition in a number of ways, acting rapidly through catecholamine's and more slowly by glucocorticoids ${ }^{12}$. During stress, there is activation of sympatho-adrenal system which result in increased secretion of epinephrine (EPI) and nor epinephrine (NE) and ${ }^{13}$ activation of hypothalamicpituitary-adrenal (HPA) axis resulting in release of Corticotropin-Releasing Factor (CRF). The CRF in turn stimulates Adrenocorticotropin (ACTH) release from pituitary gland leading to the secretion of stress hormones from the adrenals. There are two main stress hormones, the glucocorticoids (called cortisol) and the catecholamines ${ }^{14}$. Cortisol is the principal glucocorticoid involved in the stress response ${ }^{16}$.

Pathways which play role in combating the stress may be divided into different types according to the time duration as; immediate stress reactions - occur within seconds, intermediated stress reactions - also within seconds but more than immediate effects and prolonged stress reactions - may exist for days, weeks, months and or years. It is hypothesized that the cognitive function of first year, second year and third year students is impaired on exposure to examination stress ${ }^{12}$.

Objective of study: To determine, by using Stroop test, the effects of mental stress upon cognitive function among medical students of Liaquat University of Medical and Health Sciences, Jamshoro.

\section{METHODOLOGY}

This cross-sectional comparative study was conducted after approval from Ethical review committee of LUMHS, from August 2015 to February 2016 at Department of Physiology, LUMHS, Jamshoro. Study population were undergraduate student, aged between 18-24 years, of MBBS and BDS course of $1^{\text {st }}, 2^{\text {nd }}$ and $3^{\text {rd }}$ year. Those having their examination within one hour were labelled as group $A$ and those having no examination were grouped as $B$. Graduates, postgraduates, students having colour blindness, psychological disorder, hypertension, diabetes, thyroid dysfunction and those taking any regular medicines were excluded. Volunteers for group B having unexplained increased pulse rate at first recording, not settling within 15 minutes were also excluded. Sample size calculated using general formula with $11 \%{ }^{17}$ prevalence, using the proportion of $95 \%$ confidential interval and $5 \%$ of margin of error, the sample size stands to be $n=150$. The volunteers were divided in two groups.

Group A: Comprises of 50 male and 50 female volunteers who have their examinations in a week time.

Group B: Comprises of 25 male and 25 females volunteers who are not going to exam process at that time and found relaxed on pulse rate determination.

Simple random sampling used to enrol volunteers. Data was collected by the principal investigator, followed by data entry / transfer on the SPSS (statistical package for social sciences) version 16 data sheets for analysis. Student t-test and Chi square test were used for continuous and categorical variables respectively.

\section{Procedure}

The volunteer information flyers were posted on the main notice board of the LUMHS campus for the recruitment of the participants. Students who showed interest were recruited only after they have undergone medical screening test, conducted at laboratory of Physiology department. Those who were found medically fit, willing to be volunteer were explained, both verbally and through written information sheet, about the purpose and procedure of the study. Those given written informed consent were finally enrolled for the study

\section{Protocol}

The participants were called in the Physiology department laboratory on a prefixed date. They were asked to sit comfortably in a chair. After explaining the protocol, pulse of the volunteer was recorded manually as well as pulse transducer was attached to the terminal phalanx of middle finger of the volunteer to ascertain or exclude any anxiety state that the volunteer may be feeling, as reflected by a changed pulse rate. 
The protocol was proceeded further for volunteers of Group-A and Group-B; one end of the pulse transducer attached to the volunteer and the second end to power labT-125 AD-instrument Australia at input 1, which record the pulse continuously and automatically to indicate any sympathetic activities and was displayed rate as graphical and numerical data throughout the test duration. The Stroop test was performed on (Power lab T-125 AD-instrument Australia).

In first exercise of the Stroop test, volunteers read the color chart given on the computer screen and while the test is being performed, the principal investigator noted the time taken for that exercise. All three exercises had performed and time was noted on separate sheets. The answer sheet for each exercise was separate on which the investigator filled as the test proceeds.

\section{RESULTS}

The study included; $n=100$ medical students under Examination stress in group $A$ while in Group $B, n=50$ medical students without Examination stress / Relaxed. Comparative differences between demographic characteristics and physiological parameters of cardiovascular function such as age, weight and height of both groups were statistically non -significant as shown in graph I.

Breathing rate, heart rate, systolic and diastolic blood pressure (BP) showed statistically significant differences. Breathing rate of group, $A$ and $B$ was $14.18 \pm 1.22$ and $13.24 \pm 1.15$ per minute respectively (t- value 4.51, $\mathrm{p}$ - value 0.0001 ). Heart rate in group $A$ and $B$ was noted as $73.92 \pm 2.67$ and $71.10 \pm 1.23$ per minute respectively (t- value $7.08, \mathrm{p}$ - value 0.0001 ). Systolic BP in group A and B were noted as 124.94 \pm 8.22 and $122.28 \pm 6.19 \mathrm{mmHg}$ respectively (t- value 2.01, $p$ - value 0.046). Diastolic BP in group A and B were noted as $76.27 \pm 5.25$ and $74.14 \pm 5.38 \mathrm{mmHg}$ respectively ( $t-$ value $2.3, p$ - value 0.022 ). Breathing rate, heart rate, systolic and diastolic blood pressure (BP) is summarized in table I and graph II.

Time Domain of Exercise 1, 2, 3 and 4

Total time taken in exercise $1,2,3$, and 4 is shown in table III. Monochrome reading (exercise 1) took 27.10 \pm 1.96 and $21.82 \pm 2.06$ seconds in groups $A$ and $B$ respectively ( $p$-value 0.0001 ). Color naming (exercise 2) took $31.54 \pm 2.35$ and $24.20 \pm 3.65$ seconds in groups $A$ and $B$ respectively ( $p$-value 0.0001). Reading color words (exercise 3 ) took $34.54 \pm 2.23$ and $26.08 \pm 2.10$ seconds in groups $A$ and $B$ respectively ( $p$-value 0.0001 ). Monochrome reading (exercise 4) took $36.79 \pm 1.88$ and $28.56 \pm 3.85$ seconds in groups $A$ and $B$ respectively ( $p$-value $0.0001)$. Graphs 3 show the time domain of exercise $1,2,3$ and 4 respectively.

Time Domain of Single word - Exercise 1,2, 3 and 4 Congruent Stroop test

Congruent Stroop test - exercise 1 and 2 showed significant differences for time domain per single word in group $A$ and $B$ respectively.

Exercise 1- Time taken per single word was noted as 0.6775 vs. 0.5455 seconds in exercise 1 (t-value 15.8, p-value 0.0001).

Exercise 2- Time taken per single word was noted as 2.1000 vs. 1.6100 seconds in exercise 2 (t-value 15.8, p-value 0.0001). Details are shown in table III and graphs 3 respectively.

\section{Incongruent stroop test}

Exercise 3- Time taken per single word was noted as 2.3000 vs. 1.7500 seconds in exercise 3 (t-value 23.13, p-value 0.0001).

Exercise 4- Time taken per single word was noted as 2.1000 vs. 1.6100 seconds in exercise 3 (t-value 16.56, p-value 0.0001). Details are shown in table I11 and graphs 3 respectively.

TABLE I: PHYSIOLOGICAL CHARACTERISTICS OF STUDY POPULATION

\begin{tabular}{|l|r|r|r|r|r|r|}
\hline & \multicolumn{2}{|c|}{ Group A(n=100) } & \multicolumn{2}{c|}{ Group B(n=50) } & \multirow{2}{*}{ t-value } & \multirow{2}{*}{ p-value } \\
\cline { 2 - 5 } & \multicolumn{1}{|c|}{ Mean } & \multicolumn{1}{c|}{ SD } & \multicolumn{1}{c|}{ Mean } & \multicolumn{1}{c|}{ SD } & & \\
\hline Age (years) & 21.63 & 1.83 & 21.46 & 1.78 & 0.541 & 0.059 \\
\hline Weight (kg) & 61.23 & 6.73 & 61.69 & 6.96 & 0.39 & 0.056 \\
\hline Height (ft) & 5.24 & 0.28 & 5.23 & 0.29 & 0.18 & 0.085 \\
\hline Breathing rate (/minute) & 14.18 & 1.22 & 13.24 & 1.15 & 4.51 & 0.0001 \\
\hline Heart rate (/minute) & 73.92 & 2.67 & 71.10 & 1.23 & 7.08 & 0.0001 \\
\hline Systolic BP (mmHg) & 124.94 & 8.22 & 122.28 & 6.19 & 2.01 & 0.046 \\
\hline Diastolic BP (mmHg) & 76.27 & 5.25 & 74.14 & 5.38 & 2.3 & 0.022 \\
\hline
\end{tabular}


Determination of Mental Stress Effects on the Cognitive Function

TABLE II: TIME TAKEN IN DIFFERENT EXERCISES BY STUDY POPULATION ( $n=150)$

\begin{tabular}{|l|r|r|r|r|r|r|}
\hline & \multicolumn{2}{|c|}{ Group A (n=100) } & \multicolumn{2}{c|}{ Group B (n=50) } & \multirow{2}{*}{ t-value } & \multirow{2}{*}{ p-value } \\
\cline { 2 - 6 } & Mean & \multicolumn{1}{c|}{ SD } & Mean & \multicolumn{1}{c|}{ SD } & & \\
\hline Exercise 1- Monochrome reading (seconds) & 27.10 & 1.96 & 21.82 & 2.06 & 15.2 & 0.0001 \\
\hline Exercise 2- Color naming (seconds) & 31.54 & 2.35 & 24.20 & 3.65 & 14.8 & 0.0001 \\
\hline Exercise 3- Reading color words (seconds) & 34.54 & 2.23 & 26.08 & 2.10 & 22.29 & 0.0001 \\
\hline Exercise 4- Color naming (seconds) & 36.79 & 1.88 & 28.56 & 3.85 & 17.59 & 0.0001 \\
\hline
\end{tabular}

TABLE III: TIME DOMAIN PER SINGLE WORD IN EXERCISE 1 TO 4 OF STUDY POPULATION ( $n=150)$

\begin{tabular}{|l|r|r|r|r|}
\hline & Group A(n=100) & Group B ( $\mathbf{n = 5 0 )}$ & \multirow{2}{*}{ t-value } & \multirow{2}{*}{$\mathbf{p}$-value } \\
\cline { 1 - 3 } & \multicolumn{1}{|c|}{ seconds } & \multicolumn{1}{c|}{ seconds } & & \\
\hline Exercise 1- Monochrome reading (seconds) & 0.6775 & 0.5455 & 15.8 & 0.0001 \\
\hline Exercise 2- Color naming (seconds) & 2.1000 & 1.6100 & 14.7 & 0.0001 \\
\hline Exercise 3- Reading color words (seconds) & 2.300 & 1.7500 & 23.13 & 0.0001 \\
\hline Exercise 4- Color naming (seconds) & 1.8390 & 1.4280 & 16.56 & 0.0001 \\
\hline
\end{tabular}

GRAPH I: PHYSIOLOGICAL CHARACTERISTICS OF STUDY POPULATION

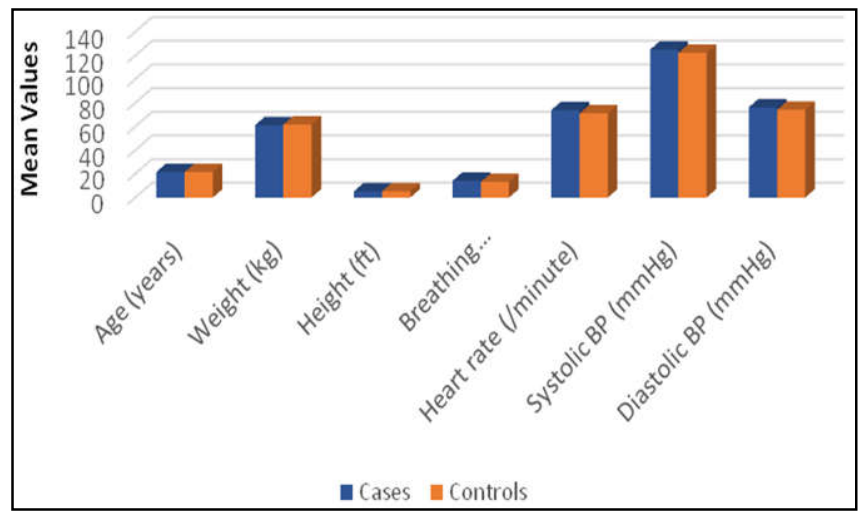

GRAPH II: TIME DOMAIN COMPARISON OF MONOCHROME READING, COLOR NAMING, READING COLOUR WORDS AND COLOUR NAMING OF GROUP A. (EXAMINATION STRESS) AND GROUP B. (NO STRESS)

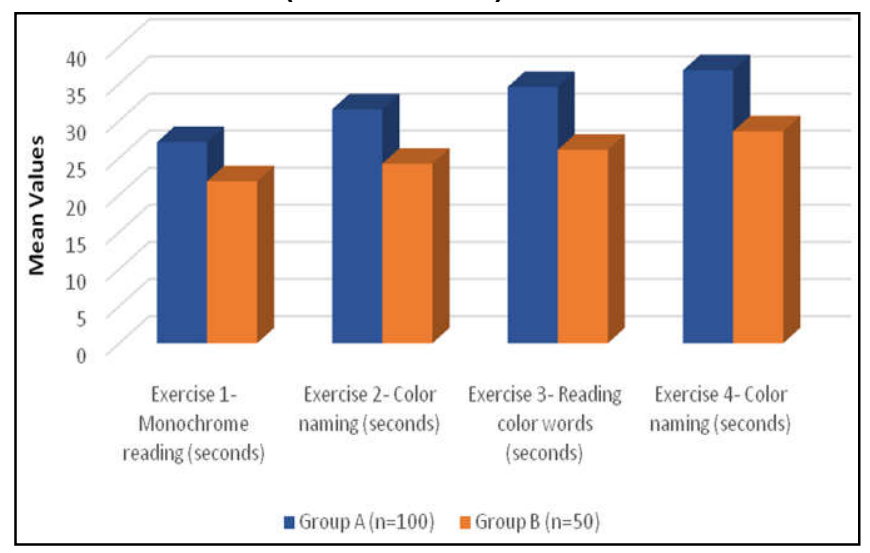

GRAPH III: TIME DOMAIN PER SINGLE WORD EXERCISE COMPARISON OF MONOCHROME READING, COLOR NAMING, READING COLOUR WORDS AND COLOR NAMING OF GROUP A. (EXAMINATION STRESS) AND GROUP B. (NO STRESS)

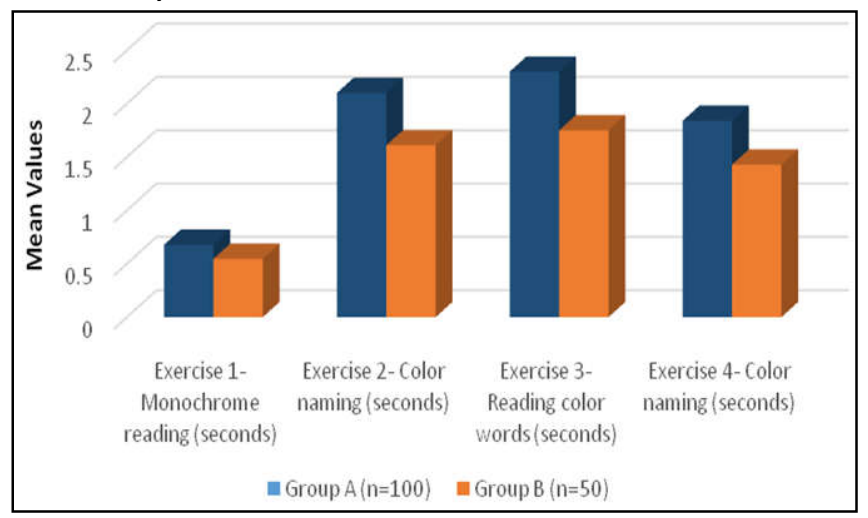

\section{DISCUSSION}

The present study is the first study on Stroop task- colour and word interference being reported from Department of Physiology, Liaquat University of Medical and Health Sciences Jamshoro, Sindh, Pakistan. Stroop test is named after its inventor the John Ridley Stroop. Since then, the Stroop task test has been used as one of the best tests available for the brain functioning under various conditions including mental stress ${ }^{19}$.

The Stroop Color-Word Test is a test used for studying the cognitive physiology and cognitive psychology. Stroop task, tests the selective attention 
under normal and stressful conditions. Interference between the meaning of word and color of ink does occur, and this prolongs the reaction time by the study subject. This occurs because they automatically encode the written words in addition to visual colors ${ }^{20}$. A previous study ${ }^{21}$ suggested that the picture and color-word Stroop task tests measure different cognitive functions. Time domain of performance during Stroop task test decreases with increasing age and with education levels; lower the education level, more time is taken ${ }^{21}$. Improvement in performance occurs with repeated practice, has been reported. Language also affects the Stroop task test timing for example Spanish versus English, but if the participants had fluency and proficiency in two or more languages, the reaction time of Stroop task test remains unaltered ${ }^{25}$.

The present study was conducted to study the effects of mental stress on cognitive functions of medical students during examinations by Stroop test. The findings of monochrome reading which was found more in stressed group A compared to relaxed group $B$ participants. Our findings of monochrome reading are consistent to previous studies ${ }^{21,22}$.

Congruent Stroop test - exercise 1 and 2 showed significant differences for time domain per single word in group $A$ and $B$ respectively.

Exercise 1- Time taken per single word was noted as 0.6775 vs. 0.5455 seconds in exercise $1(p=0.0001)$.

Exercise 2- Time taken per single word was noted as 2.1000 vs. 1.6100 seconds in exercise $2(p=0.0001)$.

Exercise 3- Time taken per single word was noted as 2.3000 vs. 1.7500 seconds in exercise $3(p=0.0001)$.

Exercise 4- Time taken per single word was noted as 2.1000 vs. 1.6100 seconds in exercise $3(p=0.0001)$.

The findings of present study showed an increase in time domain in stress persons from exercise 1 to 4 as shown above. The findings are comparable to previous studies ${ }^{21-24}$. Ghimri et $\mathrm{al}^{22}$ has recently reported a study from Nepal. The study included Medical students of BPKIHS Medical College. A classical English version of Stroop task test was used like present study. In present study, the students who knew English language were selected as by Ghimri et al ${ }^{22}$. The findings of present study are comparable to a recently reported study of Gul et $\mathrm{al}^{23}$ examined the basic Stroop effect during task performance. It was reported that the word versus color comparisons showed normal timings $(p>0.05)$ while the congruent and incongruent Stroop effects showed statistically significant differences $(p<0.05)$. Plenger et $\mathrm{al}^{25}$ evaluated the neural changes during Stroop task in 13 healthy controls and 14 patients. The Stroop effect was increased in patients with disease which indicated the frontal lobe inefficiency. The finding of Plenger et al is incomparable as regards the study subjects but the Stroop effect finding is consistent finding to the present study ${ }^{25}$. Karthikeyan et $\mathrm{al}^{24}$ assessed Stroop color word test (stressor) for induction of stress and consequently the electrocardiogram and heart rate variability were detected. Significant changes between normal and stressed states were noted $(p<0.05)$.

The outcome of present research work is helpful to develop Stroop based effects during stress and its effects on the normal physiology.

\section{CONCLUSION}

The present study showed increased reaction time and interferences for Stroop effect in medical students under examination stress. The mental stress adversely affects the cognitive functions as evaluated in present study. The reaction time was comparably more in incongruent Stroop test than congruent Stroop test.

\section{RECOMMENDATION}

Further studies are recommended to confirm the findings of present study and emphasis other factors which effects on students during examination.

\section{ACKNOWLEDGEMENT}

Authors would like to thanks to participant / students who cooperated before examination and lab staff of physiology department.

\section{REFERENCES}

1. Firth J. Levels and sources in medical students. Br Med J (Clin Res ED). 1986; 292 (6529): 1177-80

2. Seth GS. A study of stress in medical students at Seth G.S. Medical College Mumbai. J Postgrad Med 1998; 44(1):1-6.

3. Abdulghani HM., Alkanhal AA, Mahmoud E.S, Ponnamperuma GG, Alfaris E. Stress and Its Effects on Medical Students: A Cross-sectional Study at a College of Medicine in Saudi Arabia, J Health Popul Nutr. 2011; 29(5): 516-22.

4. Sharifirad G, Marjani A, Abdulrahman C, Mostafa Q, Hossein S. Stress among Isfahan medical sciences students. J Res Med Sci. 2012; 17(4): 402-6.

5. Park J, Chung S, Hoyoung An, Park S, Lee C, Kim SY, et al. A Structural Model of Stress, Motivation, and Academic Performance in Medical Students, Psychiatry Investing. 2012; 9(2): 143-9.

6. Blanch DC, Hall JA, Roter DL, Frankel RM. Medical student gender and issues of confidence. Patient Educ Counc. 2008; 72(3):374-811.

7. Backović DV, Zivojinovic J, Maksimovic J, Maksimovic M. Gender differences in academic 
stress and burnout among medical students in final years of education. Psychiatria Danubina 2012; 24(2):175-181.

8. Styles WM. Stress in undergraduate medical education: 'the mask of relaxed brilliance' $\mathrm{Br} \mathrm{J}$ Gen Pract. 1993; 43(367):46-7.

9. Hange D, Mehlig K, Lissner L, Guo X, Bengtsson C, Skoog I, et al. Perceived mental stress in women associated with psychosomatic symptoms, but not mortality: Observations from the population study of women in Gothenburg, Sweden. Int J Gen Med. 2013; 6:307-315.

10. Memon M, Macdonald I, Bennett T. Effect of mental stress on cardiovascular function at rest and after ingestion of fructose or sucralose in healthy, white European males. Turk J Med Sci. 2013; 43:913-18.

11. Cruz SY, Fabian C, Pagan I, Rios JL, Gonzalez AM, Betancourt J, et al. Physical Activity and its Associations with Socio-demographic Characteristics, Dietary Patterns, and Perceived Academic Stress in Students Attending College in Puerto Rico. P R Health Sci J. 2013; 32(1):44-50.

12. McEwen BS, Sapolsky RM. Stress and cognitive function. Curr Opin Neurobiol. 1995; 5(2):205-16.

13. Het S, Schoofs D, Rohleder N, Wolf OT. Stress-Induced Cortisol Level Elevations Are Associated With Reduced Negative Affect After Stress: Indications for a Mood-Buffering Cortisol Effect. Psychosomatic Medicine. 2012; 74:23-32

14. Lupien SJ. Stress and Cognition. In: Binder MD, editor. Encyclopedia of Neuroscience. Germany: Uwe Windhorst Göttingen. 2009. Pp. 445-450.

15. Piazza JR, Almeida DM, Dmitrieva NO, Klein LC. Frontiers in the Use of Biomarkers of Health in Research on Stress and Aging. J Gerontol B Psychol Sci Soc Sci. 2010; 65(5):513-25.

16. MacLaughlin BW, Wang D, Noone A, et al. Stress Biomarkers in Medical Students Participating in a
Mind Body Medicine Skills Program. Evid Based Complement Alternat Med. 2011; 2011:950461.

17. World Health Organization. Mental Health Atlas 2011. WHO. Available from: http://www.who.int/ mental_health / publications/ mental_health_atlas_2011/en/

18. Khng $\bar{K} H$, Lee $K^{-}$. The Relationship between Stroop and Stop-Signal Measures of Inhibition in Adolescents: Influences from Variations in Context and Measure Estimation. PLoS ONE 2014; 9(7): e101356.

19. Cain SW, Silva EJ, Chang AM, Ronda JM, Duffy $\mathrm{JF}$. One night of sleep deprivation affects reaction time, but not interference or facilitation in a stroop task. Brain Cogn 2011; 76(1):37-42.

20. Zalonis I, Christidi F, Bonakis A, Kararizou E, Triantafyllou NI, Paraskevas G, et al. The stroop effect in Greek healthy population: normative data for the Stroop Neuropsychological Screening Test. Arch Clin Neuropsychol 2009; 24(1):81-88.

21. Dixit A, Mittal $T$. Executive functions are not affected by 24 hours of sleep deprivation: A color-word stroop task study. Indian J Psychol Med. 2015; 37(2):165-8.

22. Ghimire N, Paudel BH, Khadka R, Singh PN. Reaction time in Stroop test in Nepalese Medical Students. J Clin Diag Res 2014; 8 (9): BC14BC16.

23. Gul A, Humphreys GW. Practice and color word integration in Stroop interference. Psicológica 2015;36: 37-67.

24. Karthikeyan P, Murugappan M, Yaacob S. Analysis of Stroop Color Word Test-Based Human Stress Detection using Electrocardiography and Heart Rate Variability Signals. AJSE. 2014; 39 (3):1835-47.

25. Plenger $P$, Krishnan $K$, Cloud $M$, Bosworth $C$, Qualls D, de la Plata CM. fNIRS-based investigation of the Stroop task after TBI. Brain Imaging Behav. 2016; 10(2):357-66.

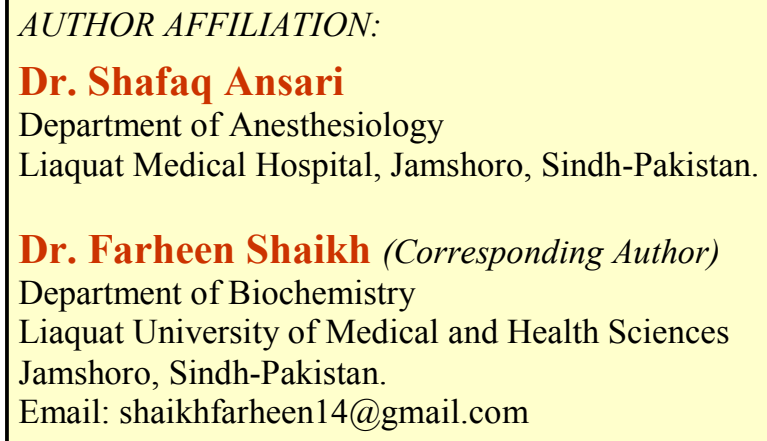

\author{
Dr. Tazeen Shah \\ Department of Physiology \\ Liaquat University of Medical and Health Sciences \\ Jamshoro, Sindh-Pakistan.
}

